\title{
COMMENTARY
}

\section{AHL-driven quorum-sensing circuits: their frequency and function among the Proteobacteria}

\author{
Rebecca J Case, Maurizio Labbate and Staffan Kjelleberg
}

The ISME Journal (2008) 2, 345-349; doi:10.1038/ ismej.2008.13; published online 14 February 2008

It is now apparent that bacteria utilize regulatory systems called quorum sensing (QS) to sense their population density. Such systems are dependant on the production of signaling molecules that activate specific genes when the signal reaches a critical threshold concentration. Such QS-regulated genes produce phenotypes that require coordinate behavior to convey competitive advantage to the population (such as biofilm formation and pathogenesis). The best-characterized QS system is that driven by acylated homoserine lactone (AHL) molecules.

Quorum sensing-regulated phenotypes are diverse; however, their evolutionary selection is based on the competitive advantage conveyed by coordinating gene expression with the establishment of a quorum. Population density and coordinated gene expression are coupled for either (1) the multicellular characteristic of behaviors such as cell differentiation (for example swarming, biofilm formation), or (2) the fitness benefit of many individual cells simultaneously expressing the same phenotype (for example virulence factors, luminescence). QS enables a population to differentiate under favorable conditions where the population is dense enough to support the division and coordination of labor into subpopulations. In undifferentiated populations, QS coordinates gene expression so that it is simultaneous for cells within the population.

In both scenarios, having QS regulation provides a competitive advantage for a population to both produce and respond to QS molecules. A selective pressure also exists for non-QS bacteria to sense and respond to QS molecules produced within the community. Examples of QS bacteria and bacteria able to detect and respond to exogenous signals are found in the literature; however, the frequency of QS and QS cheaters in the environment is poorly documented. With the growing number of bacterial genomes sequenced, especially genomes of nonclinically isolated bacteria, it may not be surprising that the number of genomes containing homologs of AHL-QS circuitry is ever growing. In this article, we use all current bacterial genomes to examine the frequency of AHL-QS among these bacteria, and the surprising number of bacteria with the genetic potential for eavesdropping on AHL signals from other bacteria.

\section{Frequency of QS among genome- sequenced bacteria}

The AHL-QS system is characterized by two core proteins, the LuxI-type protein (the AHL synthase), and the LuxR-type protein (the response regulator). Almost all AHL-producing bacteria rely on only these two protein families for active QS circuits; however, exceptions exist such as the LuxM AHL synthase in Vibrio harveyi and the putative HdtS AHL synthase identified in Pseudomonas fluorescens F113, both of which replace the LuxI-type AHL synthase.

Using the 512 completed bacterial genomes as a data set (http://www.ncbi.nlm.nih.gov/sutils/genom_table.cgi on 26 July 2007), LuxI and LuxR homologs were found only within proteobacterial genomes. Complete QS circuits (that is, containing at least one LuxI and LuxR homolog) were identified from $68(13 \%)$ of the bacterial genomes, or $26 \%$ of the 265 proteobacterial genomes (Table 1). When compared to metagenomic data, these statistics seem high. The predicted proteome of the marine metagenome (Rusch et al., 2007) recovered 20 LuxI and 31 LuxR homologs, and a 130-genome equivalent metagenomic library of Alaskan sediments recovered a single clone capable of inducing an AHL reporter and containing a luxI homolog (Williamson et al., 2005). However, all of these figures are biased, for instance whole-organism genomics rely on culturing, there is a sampling bias of filtered seawater used to construct the marine metagenome (mainly free-living bacteria are represented in the metagenome), there are limitations in expressing environmental DNA from metagenomic libraries and AHL reporters are unable to detect the structural diversity of AHLs. Whatever be the limitations of our methodologies, the number of bacteria in which we identify QS is ever increasing, 
Table 1 Bacterial genomes with AHL-driven quorum-sensing networks

\begin{tabular}{ccc}
\hline Genome & $\begin{array}{c}\text { No. of LuxI } \\
\text { homologs }\end{array}$ & $\begin{array}{c}\text { No. of LuxR } \\
\text { homologs }\end{array}$
\end{tabular}

Acidovorax avenae subsp. citrulli homologs $^{\mathrm{a}} \quad$ homologs $^{\mathrm{a}}$

AAC00-1

Acinetobacter baumannii ATCC 17978

Aeromonas hydrophila subsp. hydrophila ATCC 7966

A. salmonicida subsp. salmonicida A449

Agrobacterium tumefaciens C58

Bradyrhizobium japonicum

USDA 110

Bradyrhizobium sp. BTAi1

Bradyrhizobium sp. ORS278

Burkholderia cenocepacia AU 1054

B. cenocepacia HI2424

B. cepacia AMMD

B. mallei ATCC 23344

B. mallei NCTC 10229

B. mallei NCTC 10247

B. mallei SAVP1

B. pseudomallei 1106a

B. pseudomallei $1710 \mathrm{~b}$

B. pseudomallei 668

B. pseudomallei K96243

Burkholderia sp. 383

B. thailandensis E264

B. vietnamiensis $\mathrm{G} 4$

$B$. xenovorans LB400

Chromobacterium violaceum

ATCC 12472

Erwinia carotovora subsp.

Atroseptica SCRI1043

Geobacter uraniumreducens Rf4

Jannaschia sp. CCS1

Mesorhizobium loti MAFF303099

Mesorhizobium sp. BNC1

Nitrobacter hamburgensis X14

$N$. winogradskyi Nb-255

Nitrosospira multiformis

ATCC25196

Pseudoalteromonas atlantica T6c

Pseudomonas aeruginosa PA7

P. aeruginosa $\mathrm{PAO} 1$

P. aeruginosa UCBPP-PA14

P. syringae pv. phaseolicola $1448 \mathrm{~A}$

$P$. syringae pv. syringae $\mathrm{B} 728 \mathrm{a}$

P. syringae pv. tomato DC3000

Ralstonia solanacearum GMI1000

Rhizobium etli CFN 42

$R$. leguminosarum bv. viciae 3841

Rhodobacter sphaeroides 2.4.1

R. sphaeroides ATCC 17025

R. sphaeroides ATCC 17029

Rhodopseudomonas palustris

BisA53

R. palustris BisB18

R. palustris BisB5

R. palustris CGA009

R. palustris $\mathrm{HaA} 2$

Rhodospirillum rubrum ATCC

11170

Roseobacter denitrificans OCh 114

Saccharophagus degradans 2-40

Silicibacter pomeroyi DSS-3

Sinorhizobium medicae WSM419

S. meliloti 1021

Sodalis glossinidius morsitans
Table 1 Continued

\begin{tabular}{lcc}
\hline Genome & $\begin{array}{c}\text { No. of LuxI } \\
\text { homologs }\end{array}$ & $\begin{array}{c}\text { No. of LuxR } \\
\text { homologs }\end{array}$ \\
\hline $\begin{array}{l}\text { Sphingopyxis alaskensis RB2256 } \\
\text { Sphingomonas wittichii RW1 }\end{array}$ & $\mathbf{2}$ & $\mathbf{3}$ \\
Vibrio fischeri ES114 & $\mathbf{1}$ & $\mathbf{3}$ \\
Yersinia enterocolitica subsp. & 1 & 1 \\
Enterocolitica 8081 & $\mathbf{1}$ & $\mathbf{2}$ \\
Y. pestis Antiqua & 2 & \\
Y. pestis bv. Microtus 91001 & 2 & 2 \\
Y. pestis CO92 & 2 & 2 \\
Y. pestis KIM & 2 & 2 \\
Y. pestis Nepal516 & 2 & 2 \\
Y. pestis Pestoides F & $\mathbf{1}$ & $\mathbf{2}$ \\
Y. pseudotuberculosis IP 32953 & 2 & 2 \\
\end{tabular}

Abbreviation: AHL, acylated homoserine lactone.

aHomologs of LuxI and LuxR were identified using the following definitions: a LuxI homolog is 190-230 amino acids long and defined by a BLASTP e-value greater than $10^{-6}$ to a characterized LuxI-type AHL synthase. A LuxR homolog is 200-240 amino acids long, has a BLASTP e-value greater than $10^{-6}$ to a characterized LuxR-type, AHL response regulator and shows homology to an AHL-binding domain within 30 amino acids of its $\mathrm{N}$ terminus and homology to the GerEtype DNA-binding domain at the $\mathrm{C}$ terminus. Some two-component response regulators have DNA-binding domains homologous to the GerE-type; however, they were excluded as their ligand-binding domain lacked homology to an AHL-binding domain.

with genome sequencing a major source of this discovery.

Among the bacterial genomes in which QS is identified, several are surprising. The role of QS among several such environmental bacterial isolates is unknown and unlikely to be predicted from model QS organisms, which are generally pathogens or symbionts of a eukaryotic host. Environmental strains identified here with complete QS circuits are not known to be pathogens or symbionts; rather they play important roles in biogeochemical cycles (for example the uranium-reducing Geobacter uraniumreducens and the oligotroph Sphingopyxis alaskensis).

\section{Extra LuxR-type proteins}

The number of LuxI- and LuxR-type proteins in a given bacterium is not always equal. In fact, 45 of the $68(66 \%)$ bacteria identified here with QS circuits have more LuxR than LuxI homologs (Table 1-numbers in bold). In a QS circuit, a LuxI-type protein has a specific LuxR-type protein that binds its AHL, but there are often 'extra' LuxRtype proteins. For example, Pseudomonas aeruginosa contains two QS circuits (LasI/R and RhlI/R) but also contains QscR and a fourth LuxR-type protein identified here (Figure 1b). It has been suggested that these extra LuxR-type proteins are orphans (Fuqua, 2006); however, as they belong to a characterized protein family (AHL response regulators) and the biological role of several has been described (Wang et al., 1991; Smith and Ahmer, 
2003; Lee et al., 2006, 2007; Lequette et al., 2006), they cannot be considered orphans.

The best-characterized of these extra LuxR-type proteins in a bacterium with complete QS circuits is BisR in Rhizobium leguminosarum bv. viciae. $R$. leguminosarum bv. viciae has three QS networks, cin, rai and tra, with an additional LuxR-type protein, BisR. BisR negatively regulates cin QS, thereby maintaining a low endogenous 3O-C14-HSL concentration (as CinI synthesizes 3O-C14-HSL) (Danino et al., 2003). As 3O-C14-HSL is also the cognate AHL for BisR, BisR requires exogenous 3OC14-HSL for it to positively regulate traR, which induces conjugal transfer (Danino et al., 2003). The incorporation of BisR into the QS regulatory network allows $R$. leguminosarum bv. viciae to induce conjugal transfer only when a quorum of 3O-C14-HSL-producing bacteria (the recipients) is

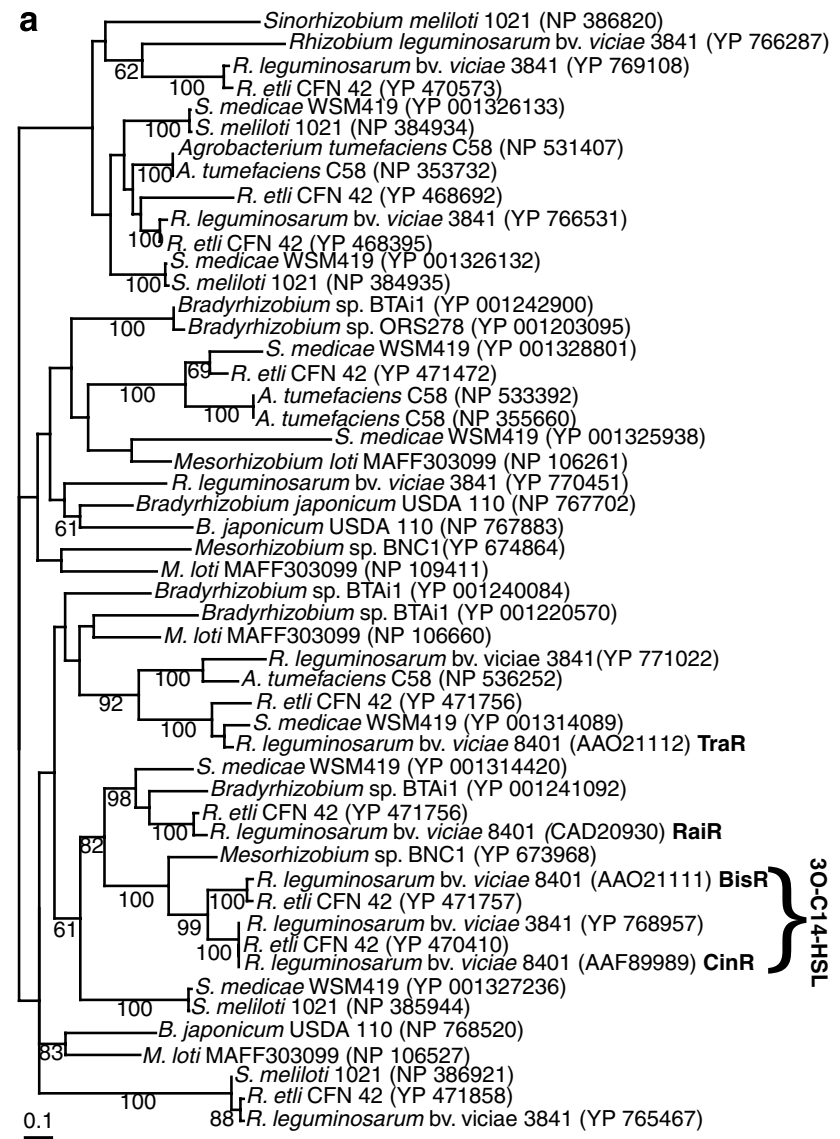

Figure 1 Predicted LuxR-type proteins from Rhizobia and Agrobacterium genomes (a) and Pseudomonas and Burkholderia genomes (b). Rhizobium leguminosarum bv. viciae 8401 is included in (a) as its AHL-QS circuits are characterized (Danino et al., 2003), although its genome is not sequenced. $P$. aeruginosa PAO1 and R. leguminosarum bv. viciae 8401 LuxR-type proteins are given in bold. The number in parentheses found after each taxon name is the accession number for the respective protein sequence. The trees were compiled by maximum likelihood using PROML. The bootstrap support value displayed above the nodes represents the consensus of maximum likelihood trees obtained from 100 pseudo-replicates of the original data set (only values above 50 are displayed).

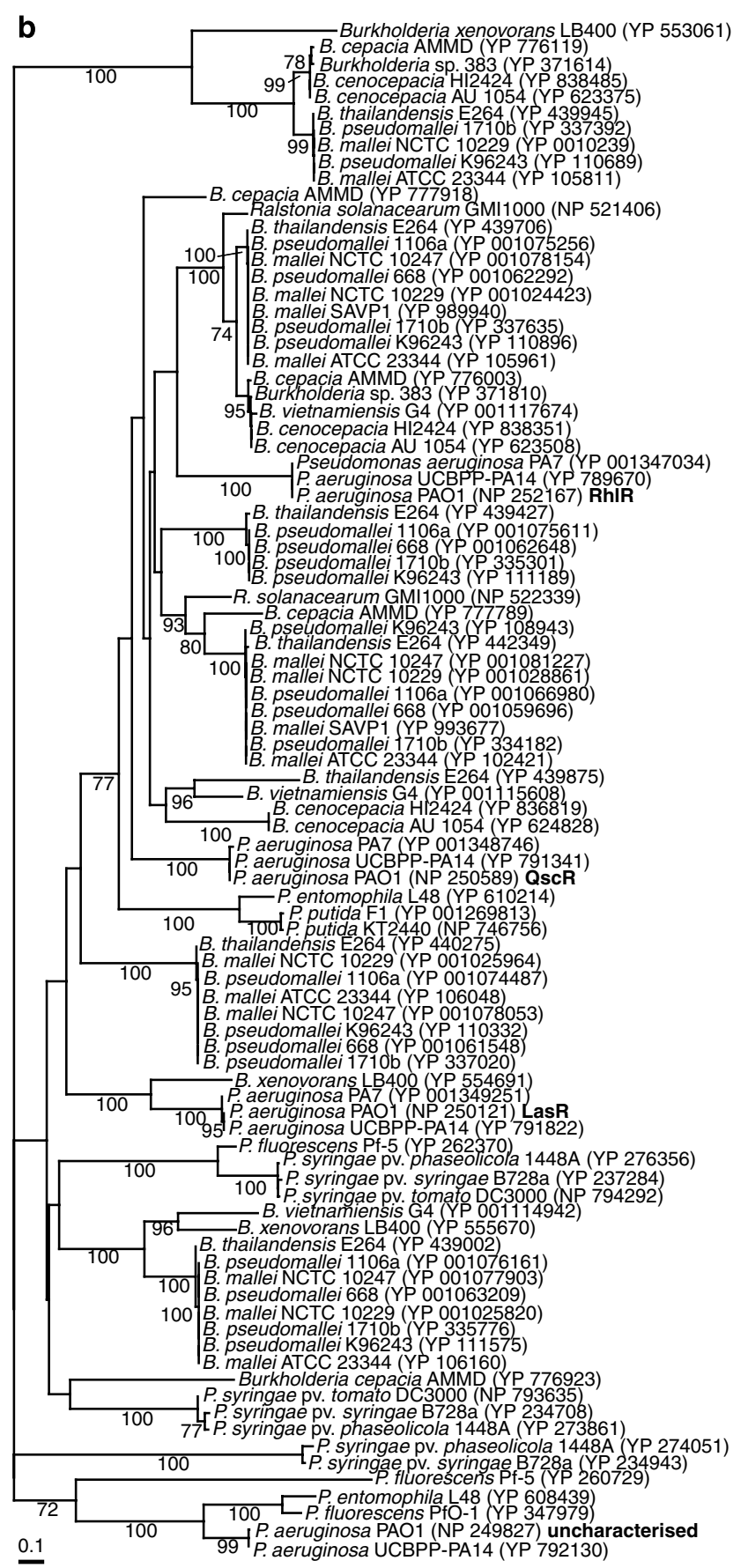

Figure 1 Continued.

established. Figure 1a illustrates the phylogeny of rhizobia and Agrobacterium LuxR-type proteins with the LuxR-type proteins whose cognate AHL is 3O-C14-HSL, forming a monophyletic group. This shows that cross talk between these species is due to the close evolutionary relationship of the 3O-C14-HSL-binding LuxR-type proteins within the rhizobia.

Another example of an uncoupled LuxR-type protein is QscR in P. aeruginosa. QscR negatively regulates both the las and rhl QS system by binding LasR, RhlR and their cognate AHLs (Ledgham et al., 


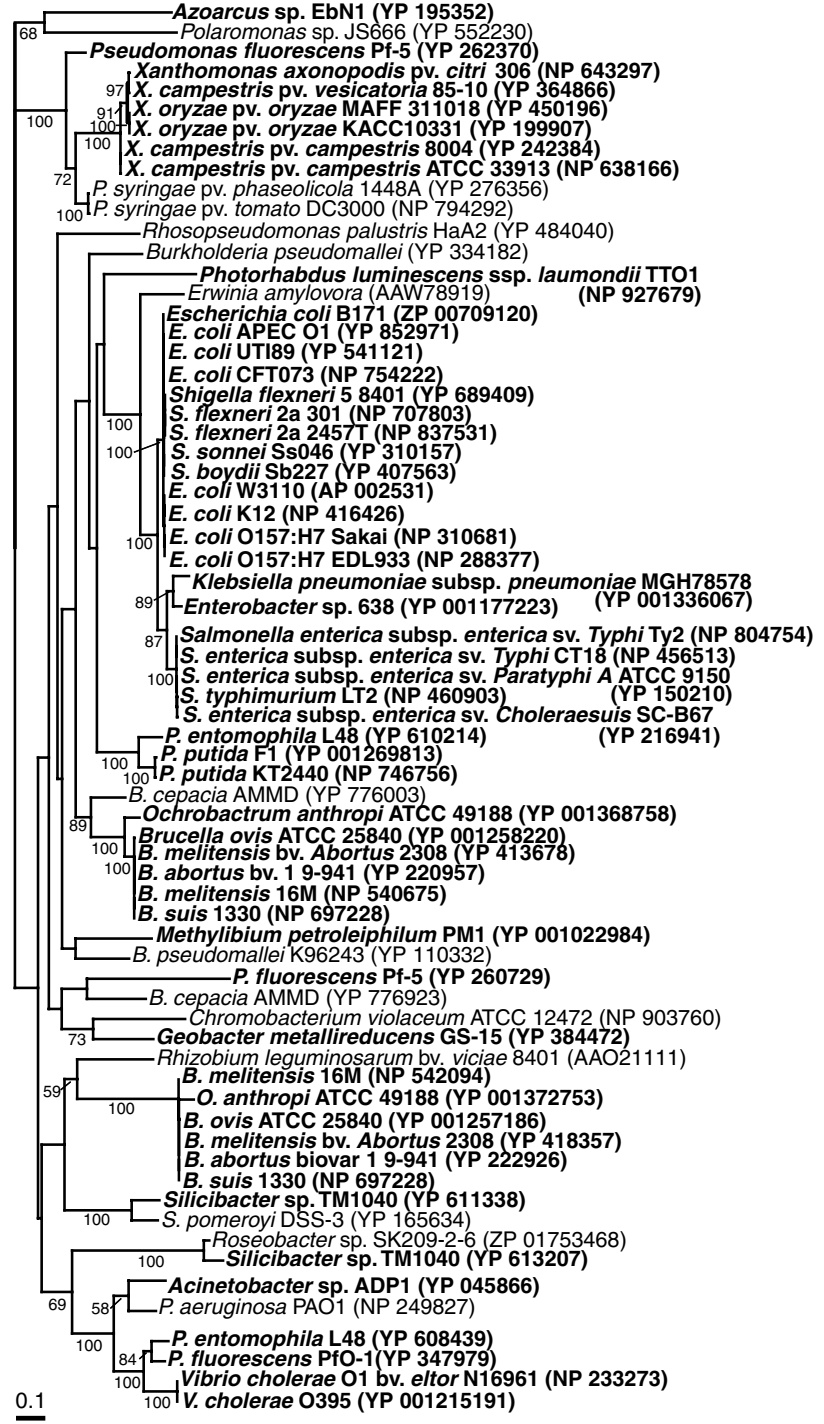

Figure 2 Predicted LuxR-type proteins from bacterial genomes that lack complete AHL-QS circuits and nearest neighbours. Fiftythree LuxR-type proteins found from 45 bacterial genomes that lack complete AHL-QS circuits are shown in bold. The number in parentheses found after each taxon name is the accession number for the respective protein sequence. The trees were compiled by maximum likelihood using PROML. The bootstrap support value displayed above the nodes represents the consensus of maximum likelihood trees obtained from 100 pseudo-replicates of the original data set (only values above 50 are displayed).

2003). QscR has been shown to affect a distinct regulon that is activated by the LasI-generated signal 3O-C12-HSL (Lequette et al., 2006). Furthermore, QscR has less specificity for AHL signals and is more sensitive to 3O-C10-HSL, an AHL not produced by $P$. aeruginosa, suggesting that QscR may function by responding to other AHLs produced by cohabitating microbes (Ledgham et al., 2003; Fuqua, 2006; Lee et al., 2006). We propose that the most-likely exogenous AHL candidates for QscR are derived from other Pseudomonas spp. and Burkholderia spp. Pseudomonas and Burkholderia have a similar life style as ubiquitous organisms commonly found in soil, and some species within these genera are opportunistic pathogens. Although they are from different classes of the Proteobacteria, LuxR-type proteins from Burkholderia spp. and Pseudomonas spp. are most related to each other, suggesting that their coding genes have been involved in lateral gene transfer (Figure 1b). This shared evolutionary history resulting from lateral transfer could facilitate cross talk between Burkholderia spp. and Pseudomonas spp.

\section{Incomplete QS circuits}

Bacteria exist that contain a LuxR but not LuxI homolog (that is, lacking complete QS circuits) such as Brucella melitensis, Salmonella typhimurium and Escherichia coli. S. typhimurium and E. coli have a luxR homolog called sdiA, but do not produce any detectable AHLs (Wang et al., 1991; Smith and Ahmer, 2003). Importantly, SdiA has been shown to respond to exogenous AHLs and regulate virulence in S. typhimurium and biofilm formation in E. coli (Wang et al., 1991; Smith and Ahmer, 2003; Lee et al., 2007); both phenotypes are important for competition and host interactions.

Why would bacteria without QS contain luxR homologs in their genome? These luxR homologs are unlikely to be redundant remnants of gene acquisition or loss as none of the completed bacterial genomes contain only a luxI homolog. One possible selective pressure to maintain these luxR homologs is for sensing and response to QS signals produced by competing bacteria. The incorporation of exogenous signals into the transcriptional regulation of genes mediating species interaction would create the potential for bacteria to eavesdrop on QS by competing bacteria and modulate the interaction with its competitor(s). Such phenotypes are regulated by SdiA.

A phylogenetic survey of all bacterial genomes in the database identified 45 bacteria that contained LuxR homolog(s) but no corresponding AHL synthase (Figure 2-sequences in bold). While it is unknown if all LuxR homologs are functional, it suggests that eavesdropping on QS bacteria may be prevalent among the Proteobacteria, with $26 \%$ of genome-sequenced Proteobacteria with complete QS circuits and another $17 \%$ with the potential to eavesdrop on AHL-driven QS in other Proteobacteria.

Given that bacteria rarely live in monoculture but rather in complex mixed communities, it would not be surprising that bacteria evolve the ability to eavesdrop. The ability to eavesdrop on the quorum established by another organism and consequently alter the transcriptome is a level of social sophistication previously not thought to exist among bacteria. $R$. leguminosarum bv. viciae eavesdrops on its 'friends' to delay conjugation until a quorum is established. Other bacteria may eavesdrop on 
'foes' to exploit their QS response (such as nutrients made available by exoenzymes).

RJ Case is at The Centre of Marine Bio-Innovation, University of New South Wales, Sydney, New South Wales, Australia; $M$ Labbate is at The Department of Chemistry and Biomolecular Sciences, Macquarie University, Sydney, New South Wales, Australia and $S$ Kjelleberg is at The Centre of Marine Bio-Innovation, University of

New South Wales, Sydney, New South Wales, Australia and The School of Biotechnology and Biomolecular Sciences, University of

New South Wales, Sydney,

New South Wales, Australia E-mail: s.kjelleberg@unsw.edu.au Current address: RJ Case is at Harvard University Center for the Environment (HUCE), 24 Oxford St, Harvard University, Cambridge, MA, USA

\section{References}

Danino VE, Wilkinson A, Edwards A, Downie JA. (2003). Recipient-induced transfer of the symbiotic plasmid pRL1JI in Rhizobium leguminosarum bv. viciae is regulated by a quorum-sensing relay. Mol Microbiol 50: $511-525$.
Fuqua C. (2006). The QscR quorum-sensing regulon of Pseudomonas aeruginosa: an orphan claims its identity. J Bacteriol 188: 3169-3171.

Ledgham F, Ventre I, Soscia C, Foglino M, Sturgis JN, Lazdunski A. (2003). Interactions of the quorum sensing regulator QscR: interaction with itself and the other regulators of Pseudomonas aeruginosa LasR and RhlR. Mol Microbiol 48: 199-210.

Lee J, Jayaraman A, Wood TK. (2007). Indole is an interspecies biofilm signal mediated by SdiA. BMC Microbiol 7: 42.

Lee JH, Lequette Y, Greenberg EP. (2006). Activity of purified QscR, a Pseudomonas aeruginosa orphan quorum-sensing transcription factor. Mol Microbiol 59: $602-609$.

Lequette Y, Lee JH, Ledgham F, Lazdunski A, Greenberg EP. (2006). A distinct QscR regulon in the Pseudomonas aeruginosa quorum-sensing circuit. J Bacteriol 188: $3365-3370$.

Rusch DB, Halpern AL, Sutton G, Heidelberg KB, Williamson S, Yooseph S et al. (2007). The sorcerer II global ocean sampling expedition: northwest Atlantic through eastern tropical Pacific. PLoS Biol 5: e77.

Smith JN, Ahmer BM. (2003). Detection of other microbial species by Salmonella: expression of the SdiA regulon. J Bacteriol 185: 1357-1366.

Wang XD, de Boer PA, Rothfield LI. (1991). A factor that positively regulates cell division by activating transcription of the major cluster of essential cell division genes of Escherichia coli. EMBO J $\mathbf{1 0}$ 3363-3372.

Williamson LL, Borlee BR, Schloss PD, Guan C, Allen HK, Handelsman J. (2005). Intracellular screen to identify metagenomic clones that induce or inhibit a quorum-sensing biosensor. Appl Environ Microbiol 71: 6335-6344. 